\title{
Winnerless competition in coupled Lotka-Volterra maps
}

\author{
L. A. González-Díaz, ${ }^{1, *}$ E. D. Gutiérrez, ${ }^{1}$ P. Varona, ${ }^{2}$ and J. L. Cabrera ${ }^{1, \dagger}$ \\ ${ }^{1}$ Laboratorio de Dinámica Estocástica, Centro de Física, Instituto Venezolano de Investigaciones Científicas, Caracas 1020-A, Venezuela \\ ${ }^{2}$ Grupo de Neurocomputación Biológica, Departamento de Ingeniería Informática, Universidad Autónoma de Madrid, 28049 Madrid, Spain
}

(Received 7 September 2012; revised manuscript received 14 May 2013; published 11 July 2013)

\begin{abstract}
Winnerless competition is analyzed in coupled maps with discrete temporal evolution of the Lotka-Volterra type of arbitrary dimension. Necessary and sufficient conditions for the appearance of structurally stable heteroclinic cycles as a function of the model parameters are deduced. It is shown that under such conditions winnerless competition dynamics is fully exhibited. Based on these conditions different cases characterizing low, intermediate, and high dimensions are therefore computationally recreated. An analytical expression for the residence times valid in the $N$-dimensional case is deduced and successfully compared with the simulations.
\end{abstract}

DOI: 10.1103/PhysRevE.88.012709

PACS number(s): 87.19.1j, 87.23.Cc, 05.45.-a, 89.75.-k

\section{INTRODUCTION}

In dynamical systems a heteroclinic orbit is a path in phase space joining two different equilibrium points, and a heteroclinic cycle ( $\mathrm{HC})$ is a collection of solution trajectories connecting equilibriums, periodic solutions, or chaotic invariant sets via saddle-sink connections. $\mathrm{HC}$ is a mechanism producing complex behavior in deterministic dynamical systems; i.e., heteroclinic orbits are related to recurrence, intermittency, and other nontrivial sequential dynamics. A $\mathrm{HC}$ is known as structurally stable if its topology does not change by arbitrarily small perturbations. Note that this situation should be distinguished from the case where the $\mathrm{HC}$ is maintained under the constant influence of an external perturbation without which the cycle vanishes [1-4]. It is well known that in continuous systems symmetry properties can lead to structurally or asymptotically stable HCs [5,6]. Robust HCs determine behavior in a wide range of problems, such as models of competing species in ecology [3], thermal convection [7-9], coupled oscillator networks [10], and neurodynamics [11,12], among others. While HCs in continuous symmetric systems of differential equations are well documented, there are few works dealing with the analysis [13,14] and applications [15] of symmetric systems of difference equations. Symmetric systems of difference equations could help the simulation of numerous coupled units without integration errors and with minimal computational costs. For instance, this may be the case for simulations of large multispecies population dynamics or the simulation of nervous network structures in neurocomputation. HCs sustain the concept of cyclic dominance or winnerless competition (WLC) where no competing agent wins; i.e., all participant agents alternate sequentially in time [16-18]. This concept can be used to explain and implement robust sequential activity (yet sensible to informative perturbations) and has been applied to describe spatiotemporal sensory encoding [18], robust rhythm generation [19], and cognitive

\footnotetext{
*lagonzalezd@gmail.com

†juluisca@gmail.com
}

phenomena relying on transient dynamics such as working memory and decision making, including pathological states [20-22]. As WLC map models are more computationally efficient and easy to realize in hardware configurations, this type of description is also preferred for robotic applications $[23,24]$.

\section{DISCRETE CANONICAL LOTKA-VOLTERRA MODEL: GENERALITIES}

We have already emphasized the general scope of WLC dynamics. The present work considers a set of Lotka-Volterra difference equations with a functional form similar to those used in neurodynamics studies of the mollusk Clione limacina $[4,25]$. Such a term corresponds to the self-regulation present in the original works on the time continuous version in [26,27]. Closely related difference equations have been considered in population dynamics [28] and other contexts [29]. The LotkaVolterra difference equations are given by the set of maps on the unit interval

$$
\begin{aligned}
a_{i}(t+1) & =r a_{i}(t)\left(1-\sum_{j=1}^{N} \rho_{i j} a_{j}(t)\right) \\
& \equiv a_{i}(t) f_{i}\left(a_{j}(t)\right)
\end{aligned}
$$

with $\rho_{i j} \geqslant 0, \rho_{i j}=1 \forall i=j$, and $r>0$ being a control parameter. The system (1) has one interior fixed point $\mathbf{a}_{\mathbf{0}}^{*}=$ $(0,0, \ldots, 0) \in \mathbb{R}^{N}$ and $N$ exterior fixed points, $\mathbf{a}_{\mathbf{k}}^{*} \in \mathbb{R}^{N}$, such that the $k$ th fixed point has a nonzero component $\delta$ at the $k$ th vector position, i.e.,

$$
\begin{aligned}
& \mathbf{a}_{\mathbf{k}}^{*}=\left(a_{k_{1}}^{*}, \ldots, a_{k_{i}}^{*}, \ldots, a_{k_{N}}^{*}\right), \\
& a_{k_{i}}^{*}=\delta \text { for } i=k, \quad a_{k_{i}}^{*}=0 \text { for } i \neq k,
\end{aligned}
$$

with $\delta=1-\frac{1}{r}$. Therefore $\mathbf{a}_{\mathbf{1}}^{*}$ has a nonzero component $\delta$ in the first position, $\mathbf{a}_{2}^{*}$ in the second position, and so on. In each fixed point, $f_{i}\left(a_{k_{i}}^{*}\right)=1$. Linear stability analysis is carried out by calculating the Jacobian matrix whose elements can be 
shown to be

$$
J_{i j}\left(\mathbf{a}_{\mathbf{k}}^{*}\right)=r\left(1-2 a_{k_{i}}^{*}-\sum_{i \neq s}^{N} \rho_{i s} a_{k_{s}}^{*}\right) \delta_{i j}-r \rho_{i j} a_{k_{i}}^{*}\left(1-\delta_{i j}\right)
$$

for $i, j=1, \ldots, N$. Solving the eigenvalue problem for the Jacobian matrix evaluated on each exterior fixed point yields the corresponding eigenvalues and eigenvectors. Each exterior fixed point $\mathbf{a}_{\mathbf{k}}^{*}$ has $N$ eigenvalues,

$$
\begin{aligned}
& \lambda_{k_{i}}=2-r \text { for } i=k, \\
& \lambda_{k_{i}}=r-\rho_{i k}(1-r) \quad \forall i \neq k,
\end{aligned}
$$

$i=1, \ldots, N$. The eigenvalues (4) describe the motion transverse to the hyperplane $i$. Additionally, each exterior fixed point has $N$ eigenvectors,

$$
\begin{aligned}
& \mathbf{e}_{\mathbf{k}_{\mathbf{i}}}=\left(e_{k_{i_{1}}}, \ldots, e_{k_{i_{j}}}, \ldots, e_{k_{i_{N}}}\right), \quad e_{k_{i_{j}}}=1 \text { for } j=i, \\
& e_{k_{i_{j}}}=\frac{\rho_{k j}}{\rho_{j k}-2} \text { for } j \neq i, \quad j=k, \quad e_{k_{i_{j}}}=0 \quad \text { otherwise. }
\end{aligned}
$$

with $i=1, \ldots, N$. The fact that the spectrum of eigenvalues has the same structure at each fixed point is a consequence of the $\mathbf{a}_{\mathbf{k}}^{*}$ 's being symmetrically related under the action of the symmetry group $\mathbf{Z}_{N}$, i.e., $\mathbf{Z}_{N} \cdot \mathbf{a}_{\mathbf{k}}^{*}=\mathbf{a}_{\mathbf{k}+\mathbf{1}}^{*}$ and $\mathbf{Z}_{N} \cdot \mathbf{a}_{\mathbf{N}}^{*}=\mathbf{a}_{\mathbf{1}}^{*}$. This result is needed to assert the existence of a cycle.

The interior fixed point $\mathbf{a}_{\mathbf{0}}^{*}$ has $N$ eigenvalues,

$$
\lambda_{0_{i}}=r, i=1, \ldots, N
$$

and eigenvectors corresponding to unit vectors along each $i$ axis.

\section{NECESSARY AND SUFFICIENT CONDITIONS FOR THE APPEARANCE OF A HETEROCLINIC CONTOUR}

Necessary and sufficient conditions for the continuous counterpart of (1) in the three-dimensional case were deduced in [4]. We are not aware of previous works dealing with the determination of necessary or sufficient conditions for the $N$-dimensional discrete case as considered in this work. Given system (1), we are interested in determining necessary and sufficient conditions for the existence of a heteroclinic cycle able to support WLC dynamics. WLC networks produce identity-temporal or spatiotemporal coding in the form of deterministic trajectories moving along heteroclinic orbits that connect saddle fixed points or saddle limit cycles. For the sake of simplicity we assume that there is a heteroclinic orbit $\Gamma_{k, k+1}$ connecting points $\mathbf{a}_{\mathbf{k}}^{*}$ and $\mathbf{a}_{\mathbf{k}+\mathbf{1}}^{*}, k=1, \ldots, N$, and $\mathbf{a}_{\mathbf{N}+\mathbf{1}}^{*} \equiv \mathbf{a}_{\mathbf{1}}^{*}$. In such a situation the interior point $\mathbf{a}_{\mathbf{0}}^{*}$ must be a repeller; i.e., their $N$ eigenvalues should satisfy

$$
\left\|\lambda_{0_{i}}\right\|=\|r\|>1, \quad i=1, \ldots, N
$$

given that solutions of the map (1) are of the form $\lambda^{t}$. Next, the heteroclinic cycle needs to be an attracting set. Therefore the exterior fixed point $\mathbf{a}_{\mathbf{k}}^{*}$ should only have one unstable direction, while all other directions should be stable. Thus, it is required that

$$
\begin{aligned}
& \rho_{i+1 i}<1 \quad \text { for } i=1, \ldots, N, \\
& i+1=1 \quad \text { if } i=N ; \quad 1<r<3, \\
& \rho_{k i} \in\left(1, \frac{r+1}{r-1}\right) \text { for } k=1, \ldots, N, \quad i=1, \ldots, N ; \\
& k \neq i+1 ; 1<r \leqslant 3 .
\end{aligned}
$$

These restrictions on the values of the parameters $\rho_{i j}$ ensure the following: (i) There are no exterior fixed points in the $i$ axis $>0,(i+1)$ axis $>0$ positive region. Because of (1), $a_{i}(t+1)<0$ if $a_{i+1}(t) \gg 1$, and a separatrix $\Gamma_{i, i+1}$ exists on the plane $P_{i, i+1}$ connecting the saddle $a_{i}^{*}$ direction to the stable $a_{i+1}^{*}$ direction, (ii) the leading direction [30] in $a_{i}^{*}$ is transversal to the $(i+1)$ axis on $P_{i, i+1}$, and (iii) the heteroclinic cycle is structurally stable [6] and attractive.

The heteroclinic contour will be a global attractor if we require real numbers $p_{1}, \ldots, p_{N}>0$, such that

$$
\sum_{i=1}^{N} p_{i} f^{i}\left(a_{k_{i}}^{*}\right)>0
$$

for each exterior fixed point $\mathbf{a}_{\mathbf{k}}^{*}$ in the contour $\partial \mathbb{R}_{+}^{N}$ [31]. Choosing $p_{1}=p_{2}=\cdots=p_{N}=1$ and evaluating (10) at each exterior fixed point $\mathbf{a}_{\mathbf{k}}^{*}$, we obtain

$$
\sum_{i=1, i \neq k}^{N} \rho_{i k}<N \frac{r}{r-1}, \quad k=1, \ldots, N
$$

Condition (11) ensures that the region inside the heteroclinic contour repels the orbits. Because of the attractiveness of the cycle these orbits will be trapped by the heteroclinic contour only. Conditions (8), (9), and (11) on the values of the parameters $\rho_{i j}$ are necessary and sufficient conditions for the appearance of a stable heteroclinic channel (SHC) [32] with independence of the initial conditions. From now on, the matrix whose inputs are the parameters $\rho_{i j}$ satisfying (8), (9), and (11) will be called the connectivity matrix of (1).

\section{TESTING THE ANALYTICAL CONDITIONS WITH NUMERICAL SIMULATIONS}

In order to test the above analytical approach, we simulate cases with different dimensions: a low-dimensional case with $N=9$ (Fig. 1, left), an intermediate-dimensional case with $N=27$ (Fig. 1, middle), and a high-dimensional case with $N=729$ (Fig. 1, right). From now on we designate the parameter $t$ as time, even though it is dimensionless. The structure of the connectivity matrix $\hat{\rho}$ for the low-dimensional case $(N=9)$ and parameter value 


$$
r=1.5 \text { is } \hat{\rho}=\left(\begin{array}{ccccccccc}
1 & 1.15 & 1.017 & 1.015 & 1.017 & 1.015 & 1.017 & 1.015 & 0 \\
0 & 1 & 1.33 & 1.017 & 1.015 & 1.017 & 1.015 & 1.017 & 1.015 \\
1.017 & 0 & 1 & 1.28 & 1.017 & 1.015 & 1.017 & 1.015 & 1.017 \\
1.015 & 1.017 & 0 & 1 & 1.19 & 1.017 & 1.015 & 1.017 & 1.015 \\
1.017 & 1.015 & 1.017 & 0 & 1 & 1.43 & 1.017 & 1.015 & 1.017 \\
1.015 & 1.017 & 1.015 & 1.017 & 0 & 1 & 1.26 & 1.017 & 1.015 \\
1.017 & 1.015 & 1.017 & 1.015 & 1.017 & 0 & 1 & 1.17 & 1.017 \\
1.015 & 1.017 & 1.017 & 1.015 & 1.017 & 1.015 & 0 & 1 & 1.21 \\
1.37 & 1.017 & 1.015 & 1.017 & 1.015 & 1.017 & 1.015 & 0 & 1
\end{array}\right) .
$$

Cases with larger dimension were simulated using a connectivity matrix with a similar structure to (12), also satisfying conditions (8), (9), and (11) and values of the control parameter as specified in the figure captions.

In Fig. 1 the time intervals where each unit is active and in the fixed point are displayed for each value of $i$. The switching dynamics of each $a_{i}(t)$ is characteristic of WLC where only one of the solutions satisfies $a_{i}(t+1)=a_{i}(t) \neq 0$ when active. Note that the solution's qualitative behavior is the same for the different dimensions considered and, in particular, for the intermediate- and high-dimensional cases. As the dimension is increased, temporal patterns emerge in a specific sequential order.

For the parameter values used in Fig. 1, WLC is obtained in the absence of an external perturbation. So we next consider the case of the system evaluated in a set of parameters where WLC is not observed, i.e., a case where, after some initial transient, the switching dynamics stops abruptly and the trajectory is trapped in one fixed point (Fig. 2, top). Now, with the same set of parameters a constant perturbation (equal to $55 \%$ of the fixed point value, i.e., 0.275) is added to Eq. (1). A switching behavior characteristic of WLC is recovered (Fig. 2, bottom), and each $a_{i}(t)$ remains active during a short residence time. Therefore a single constant perturbation acts on the discrete version as it does on the time continuous version. The robustness of the sequence order and the flexibility in the residence times makes such a stable $\mathrm{HC}$ a good candidate for the neuronal encoding of trajectories [21]. Furthermore, the attractor of the perturbed orbit remains in a small neighborhood of the unperturbed attractor [4], i.e., in a stable heteroclinic channel [21].

\section{RESIDENCE TIMES}

To better understand system (1) we analytically study the behavior of $\Delta$, the sojourn or residence times, i.e., the time the trajectory spends around a saddle. Without loss of generality let's start considering system (1) with a $N \times N$ connectivity matrix with the following particular structure:

$$
\hat{\rho}=\left(\begin{array}{cccccc}
1 & \rho_{12} & \ldots & \rho_{1 N-2} & \rho_{1 N-1} & \rho_{1 N} \\
\rho_{1 N} & 1 & \rho_{12} & \ldots & \rho_{1 N-2} & \rho_{1 N-1} \\
\rho_{1 N-1} & \rho_{1 N} & 1 & \rho_{12} & \ldots & \rho_{1 N-2} \\
\vdots & \vdots & \vdots & \vdots & \vdots & \vdots \\
\rho_{13} & \ldots & \ldots & \rho_{1 N} & 1 & \rho_{12} \\
\rho_{12} & \ldots & \ldots & \rho_{1 N-1} & \rho_{1 N} & 1
\end{array}\right)
$$

and let's define

$$
\begin{aligned}
S(t+1) & =a_{1}(t+1)+a_{2}(t+1)+\cdots+a_{N}(t+1) \\
& =r S(t)-r \sum_{k=1}^{N} a_{k}(t)-\sum_{k=2}^{N} \rho_{1 k} \sum_{\substack{i=1 \\
i \neq j}}^{N} \sum_{\substack{j=1 \\
j \neq j}}^{N} \frac{a_{i} a_{j}}{2} .
\end{aligned}
$$
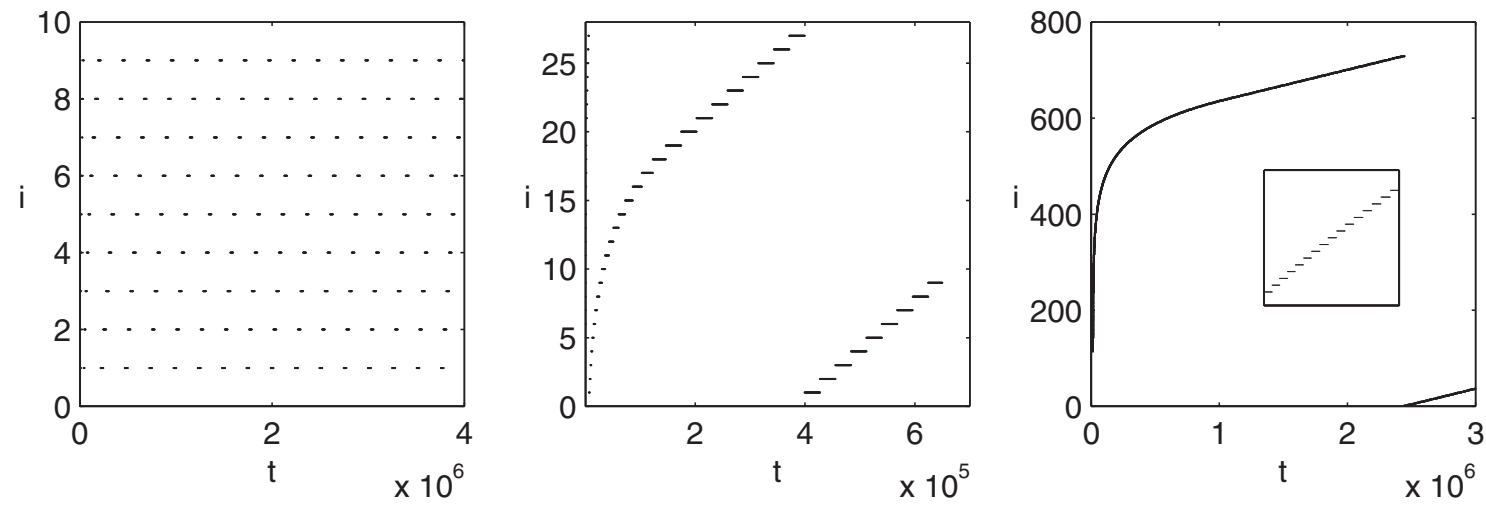

FIG. 1. Time series showing the switching process for each solution of (1). Data obtained with (left) $r=1.5$ and $N=9$, (middle) $r=1.5$ and $N=27$, and (right) $r=2.1$ and $N=729$. The inset is a temporal window showing detailed activity of units 187 to 207 . 


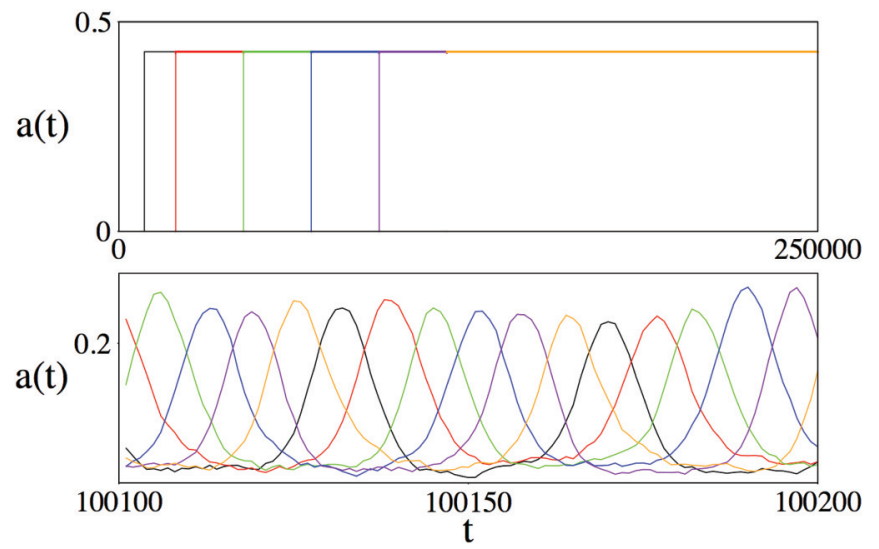

FIG. 2. (Color online) Time evolution for all units of system (1) with $N=6$ obtained with $r=2.2$, (top) with no perturbation added and (bottom) with a constant perturbation added equal to $55 \%$ of $a^{*}$, i.e., 0.275. Different colors indicate different $a_{i}(t)$.

In the vicinity of the fixed point $\mathbf{a}_{1}^{*}=(\delta, 0, \ldots, 0)$, the trajectory satisfies $a_{1}(t) \approx \delta$ and $a_{2}(t) \approx a_{3}(t) \approx \ldots \approx a_{N}(t) \approx 0$. Therefore (14) is reduced to

$$
S(t+1) \approx r S(t)[1-S(t)],
$$

whose solution is $S(t)=\delta$. Now, define the quantity

$$
\begin{aligned}
\mathcal{P} & \equiv \frac{a_{1}(t+1)}{a_{1}(t)}+\frac{a_{2}(t+1)}{a_{2}(t)}+\cdots+\frac{a_{N}(t+1)}{a_{N}(t)} \\
& =N r-r\left(1+\sum_{k=2}^{N} \rho_{i k}\right) S(t) .
\end{aligned}
$$

Evaluating this expression in the simplex, i.e., $S(t) \approx\left|\mathbf{a}_{\mathbf{1}}^{*}\right|=$ $\delta=1-\frac{1}{r}$, we obtain

$$
\mathcal{P} \approx 1+r(N-1)-\sum_{k=2}^{N} \rho_{1 k}(r-1) .
$$

For $t$ large enough and close to the saddle the trajectory satisfies $\frac{a_{1}(t+1)}{a_{1}(t)} \approx 1$ and $\frac{a_{2}(t+1)}{a_{2}(t)} \approx \ldots \approx \frac{a_{N}(t+1)}{a_{N}(t)} \equiv \mathcal{A}$. Therefore from the definition of $\mathcal{P}^{a_{1}(t)}$

$$
\mathcal{P} \approx 1+(N-1) \mathcal{A} .
$$

The value of $\mathcal{A}$ can be obtained by matching (17) and (18):

$$
1+(N-1) \mathcal{A} \approx 1+r(N-1)-\sum_{k=2}^{N} \rho_{1 k}(r-1),
$$

and $\mathcal{A}$ turns out to be

$$
\mathcal{A} \approx r-\frac{(r-1)}{N-1} \sum_{k=2}^{N} \rho_{1 k} .
$$

The trajectory approaches $\mathbf{a}_{\mathbf{1}}^{*}$ from the $\mathbf{a}_{\mathbf{N}}^{*}$ leading direction, and we just said that $\frac{a_{N}(t+1)}{a_{N}(t)} \equiv \mathcal{A}$; then,

$a_{N}(t+1) \approx \mathcal{A} a_{N}(t)=\left(r-\frac{(r-1)}{N-1} \sum_{k=2}^{N} \rho_{1 k}\right) a_{N}(t)$,

and thus the $a_{N}$ temporal evolution is given approximately by

$$
a_{N}(t) \approx\left(r-\frac{(r-1)}{N-1} \sum_{k=2}^{N} \rho_{1 k}\right)^{t} a_{N}(0)
$$

However, in the vicinity of the fixed point the system's temporal evolution is determined by the eigenvalue given by (4), i.e.,

$$
a_{N}(t) \approx\left[r\left(1-\rho_{N 1} \delta\right)\right]^{t}=\left[r-(r-1) \rho_{N 1}\right]^{t} .
$$

At this point we can combine (22) and (23):

$$
\left(r-\frac{(r-1)}{N-1} \sum_{k=2}^{N} \rho_{1 k}\right)^{t} a_{N}(0) \approx\left[r-(r-1) \rho_{N 1}\right]^{t_{\text {in }}},
$$

where $t_{\text {in }}$ is the time spent approaching the neighborhood of $(\delta, 0, \ldots, 0)$ and $t$ is the total time elapsed up to that stage. Thus,

$$
t_{\text {in }} \approx \frac{\ln a_{N}(0)}{\ln \left[r-(r-1) \rho_{N 1}\right]}+\frac{\ln \left[r-\frac{(r-1)}{N-1} \sum_{k=2}^{N} \rho_{1 k}\right]}{\ln \left[r-(r-1) \rho_{N 1}\right]} t .
$$

The trajectory is expelled from the fix point in the leading direction of $\mathbf{a}_{2}^{*}$; thus following a procedure similar to that above, we can find an expression for $t_{\text {out }}$, the time spent departing from the neighborhood of $(\delta, 0, \ldots, 0)$ :

$$
t_{\mathrm{out}} \approx \frac{\ln a_{2}(0)}{\ln \left[r-(r-1) \rho_{21}\right]}+\frac{\ln \left[r-\frac{(r-1)}{N-1} \sum_{k=2}^{N} \rho_{1 k}\right]}{\ln \left[r-(r-1) \rho_{21}\right]} t .
$$

Adding (25) and (26), the time spent by the orbit in the neighborhood of the fixed point in any one cycle is obtained:

$$
\Delta=t_{\text {in }}+t_{\text {out }}=\frac{\ln \left[r-\frac{(r-1)}{N-1} \sum_{k=2}^{N} \rho_{1 k}\right] \ln \left\{\left[r-(r-1) \rho_{21}\right]\left[r-(r-1) \rho_{N 1}\right]\right\}}{\ln \left[r-(r-1) \rho_{21}\right] \ln \left[r-(r-1) \rho_{N 1}\right]} t+\mathcal{C},
$$

where $\mathcal{C}$ is a constant that depends on the initial conditions. The relationship between the entry and exit times is deduced by combining (25) and (26),

$$
t_{\text {out }}=\frac{\ln \left[r-(r-1) \rho_{N 1}\right]}{\left|\ln \left[r-(r-1) \rho_{21}\right]\right|} t_{\text {in }} .
$$

Note that this expression is similar to that deduced in [33] for lower-dimension systems and, as in that work, the sojourn time near the saddle is analogous to the continuous case [3], except that the eigenvalues are replaced by their logarithms. We must recall that this calculation has been done on the simplex and not on the heteroclinic trajectory. This implies that we expect the 


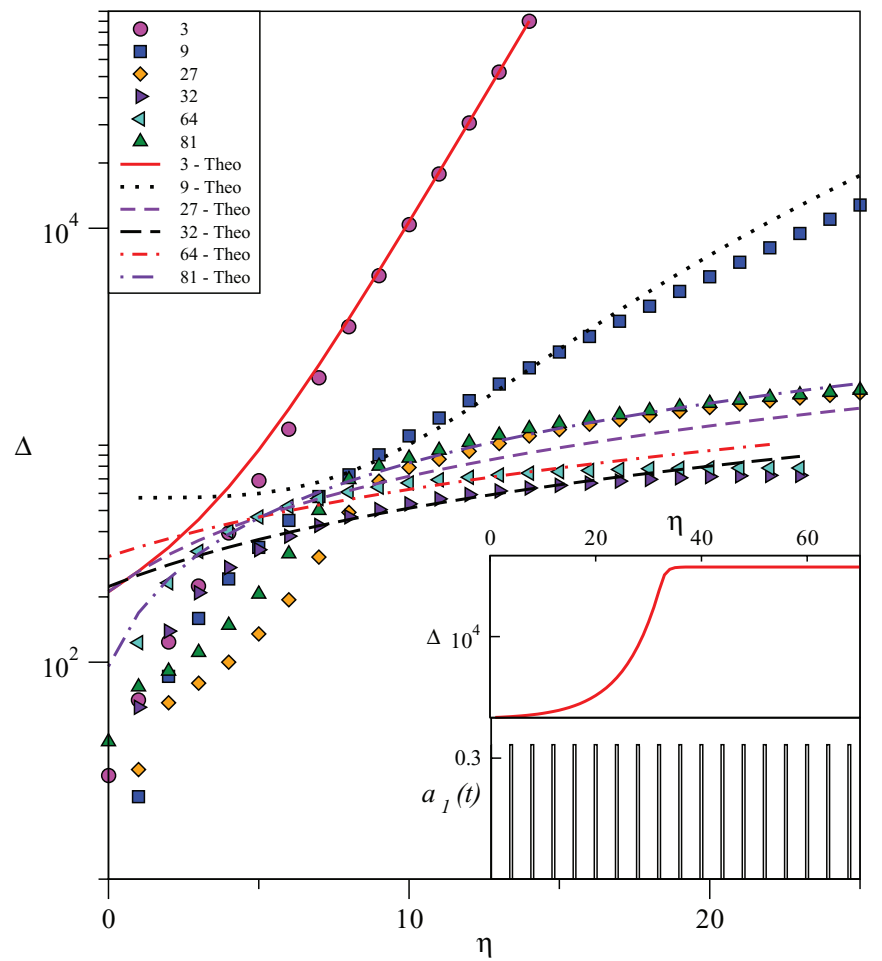

FIG. 3. (Color online) Evolution of the residence time $\Delta$ with respect to the activation number $\eta$ for $a_{1}$ in the cases with dimension $N=3$ (circles), 9 (squares), 27 (diamonds), 32 (right-pointing triangles), 64 (left-pointing triangles), and 81 (upward triangles). The top inset shows that $\Delta$ obtained with a set of parameters not satisfying (13) exhibits sustained periodic activity, as shown on the time interval $\left[5 \times 10^{6}, 7.5 \times 10^{6}\right]$ in the bottom inset.

analytical expression to approach the numerical outcomes for long times. In Fig. 3, the analytical and the numerical behaviors of $\Delta$ are plotted as a function of the activation index $\eta$ for different dimension values. For the lower-dimensional case considered $(N=3)$ the theoretical curve fits the simulation results remarkably well. For the following case shown with $N=9$ the theoretical curve still fits the experimental one pretty well. Further increasing the system dimension shows coincident trends between theory and simulation. Results are notoriously good for the case $N=81$, and theoretical curves for cases with $N=27,32,64$ also behave reasonably well. Therefore we conclude that our analytical deduction is able to describe trends of $\Delta$ for a large variety of dimensional cases. Furthermore, these results point out that for large enough dimensional situations the system tends to reach a state where the residence time behaves close to a periodical regime. This aspect was numerically analyzed in detail, revealing that before reaching such a state the trajectory is trapped in a fixed point. This trapping seem to be avoided by modifying the set of selected parameters as shown in the inset of Fig. 3, where a periodic switching between saddles is reported, at least for the times simulated. Unfortunately, the combination of parameters used does not satisfy the structure of the matrix (13); thus we cannot use the expression deduced for the sojourn times to contrast with the theory. We cannot compare our results with those for time continuous systems, as is the case with the pioneering work by May and Leonard [3], given that studies on high-dimensional situations are not available in the literature.

\section{FINAL REMARKS}

Summarizing, symmetry properties provide a way to obtain necessary and sufficient conditions for HC supporting WLC in system (1). These conditions are functions of the system control parameter $r$, which is different from continuous counterpart studies of (1), where the control parameter was fixed to 1 [4]. Our approach is valid for the $N$-dimensional situation. Numerical results illustrate WLC for low-, intermediate-, and high-dimensional cases, without decomposing the system into lower-dimensional components. This work shows WLC in high-dimensional systems; we have shown results for the competing dynamics between $3^{6}=729$ units. In the absence of external perturbations all the considered cases show self-sustained WLC. For parameter values implying no WLC an added external constant perturbation turns on the competing dynamics along a stable heteroclinic channel. An analytical expression for the times spent around the activation states which fits the simulation data reasonably well was deduced. Evidence is provided that shows that for certain matrix structures the residence time may converge to a constant value, giving place to a periodic regime over the time scales observed. Producing WLC in high-dimensional maps opens the door to massive computer simulations of neuronal systems that may have important implications for the study of dynamics based coding, sequential memory, and other important problems in neuroscience. It may also be the case for large population dynamics simulations. The consideration of flows in maps constitutes an important step to facilitate the connection between information flows in phase space with Shannon information and the capacity dimension of chaotic sets [22]. Therefore results from this work may turn out to be relevant in such a context. This contribution may have the potential to stimulate further related developments at a fundamental level or in the applied sciences.

\section{ACKNOWLEDGMENTS}

J.L.C. and E.D.G. acknowledge support from IVIC-141, L.A.G.-D. acknowledges support from IVIC-1089 and P.V. acknowledges support from MINECO TIN2012-30883.
[1] L. W. Roeger and L. J. S. Allen, J. Differ. Equations Appl. 10, 77 (2004).

[2] L. W. Roeger, Discrete Contin. Dyn. Syst., Ser. B 5, 841 (2005).
[3] R. M. May and W. Leonard, SIAM J. Appl. Math. 29, 243 (1975).

[4] V. S. Afraimovich, M. I. Rabinovich, and P. Varona, Int. J. Bifurcation Chaos 14, 1195 (2004). 
[5] M. J. Field, Trans. Am. Math. Soc. 259, 185 (1980).

[6] J. Guckenheimer and P. Holmes, Math. Proc. Cambridge Philos. Soc. 103, 189 (1988).

[7] R. M. Clever and F. H. Busse, J. Fluid Mech. 94, 609 (1979).

[8] F. H. Busse and K. E. Heikes, Science 208, 173 (1980).

[9] D. Armbruster, J. Guckenheimer, and P. Holmes, Phys. D 29, 257 (1987).

[10] A. S. Kuznetsov and J. Kurths, Phys. Rev. E 66, 026201 (2002).

[11] G. Laurent, M. Stopfer, R. W. Friedrich, M. I. Rabinovich, A. Volkovskii, and H. D. I. Abarbanel, Annu. Rev. Neurosci. 24, 263 (2001).

[12] P. Varona, R. Levi, Y. I. Arshavsky, M. I. Rabinovich, and A. I. Selverston, Neurocomputing 58, 549 (2004).

[13] A. Palacios, Int. J. Bifurcation Chaos 12, 1859 (2002).

[14] A. Palacios, J. Differ. Equations Appl. 9, 671 (2002).

[15] J. M. Casado, Phys. Rev. Lett. 91, 208102 (2003).

[16] M. Rabinovich, A. Volkovskii, P. Lecanda, R. Huerta, H. D. I. Abarbanel, and G. Laurent, Phys. Rev. Lett. 87, 068102 (2001).

[17] P. Ashwin and M. Timme, Nature (London) 436, 36 (2005).

[18] M. Rabinovich, R. Huerta, and G. Laurent, Science 321, 48 (2008).

[19] T. Nowotny and M. I. Rabinovich, Phys. Rev. Lett. 98, 128106 (2007).
[20] M. I. Rabinovich, R. Huerta, P. Varona, and V. S. Afraimovich, Biol. Cybern. 95, 519 (2006).

[21] M. I. Rabinovich, R. Huerta, P. Varona, and V. S. Afraimovich, PLoS Comput. Biol. 4, e1000072 (2008).

[22] M. I. Rabinovich, V. S. Afraimovich, C. Bick, and P. Varona, Phys. Live Rev. 9, 51 (2012).

[23] P. Arena, L. Fortuna, D. Lombardo, L. Patane, and M. G. Velarde, Int. J. Circuit Theory Appl. 37, 505 (2009).

[24] F. Herrero-Carron, F. B. Rodriguez, and P. Varona, Bioinspiration Biomimetics 6, 016006 (2011).

[25] P. Varona, M. I. Rabinovich, A. I. Selverston, and Y. I. Arshavsky, Chaos 12, 672 (2002).

[26] V. Volterra, Mem. R. Accad. Naz. Lincei 2, 31 (1926).

[27] A. Lotka, J. Wash. Acad. Sci. 22, 461 (1932).

[28] S. H. Levine, F. M. Scudo, and D. J. Plunkett, J. Math. Biol. 4, 171 (1977).

[29] Y. Morimoto, J. Phys. Soc. Jpn. 53, 2640 (1984).

[30] L. P. Shilnikov, A. L. Shilnikov, D. V. Turaev, and L. Chua, Methods of Qualitative Theory in Nonlinear Dynamics: Part I (World Scientific, Singapore, 2001).

[31] J. Hofbauer, V. Hutson, and W. Jansen, J. Math. Biol. 25, 553 (1987).

[32] M. I. Rabinovich, P. Varona, A. I. Selverston, and H. D. I. Abarbanel, Rev. Mod. Phys. 78, 1213 (2006).

[33] A. Gaunersdorfer, SIAM J. Appl. Math. 52, 1476 (1992). 